\title{
Effects of prebiotics vs a diet low in FODMAPS in patients with functional gut disorder
}

Article

Accepted Version

Including peer review corrections

Huaman, J.-W., Mego, M., Manichanh, C., Cañellas, N., Cañueto, D., Segurola, H., Jansana, M., Malalgelada, C., Accarino, A., Vulevic, J., Tzortzis, G., Gibson, G., Saperas, E., Guarner, F. and Azpiroz, F. (2018) Effects of prebiotics vs a diet low in FODMAPS in patients with functional gut disorder. Gastroenterology, 155 (4). pp. 1004-1007. ISSN 0016-5085 doi: https://doi.org/10.1053/j.gastro.2018.06.045 Available at https://centaur.reading.ac.uk/78945/

It is advisable to refer to the publisher's version if you intend to cite from the work. See Guidance on citing.

To link to this article DOI: http://dx.doi.org/10.1053/j.gastro.2018.06.045

Publisher: American Gastroenterological Association

All outputs in CentAUR are protected by Intellectual Property Rights law, including copyright law. Copyright and IPR is retained by the creators or other copyright holders. Terms and conditions for use of this material are defined in the End User Agreement. 


\section{CentAUR}

Central Archive at the University of Reading

Reading's research outputs online 


\section{Gut}

\section{EFFECT OF PREBIOTICS VERSUS LOW FODMAP DIET ON FUNCTIONAL GUT SYMPTOMS}

\begin{tabular}{|r|l|}
\hline Journal: & Gut \\
\hline Manuscript ID & gutjnl-2018-316046 \\
\hline Article Type: & Original Article \\
\hline Date Submitted by the Author: & 15 -Jan-2018 \\
\hline Complete List of Authors: & $\begin{array}{l}\text { Huaman, Jose; Hospital General de Catalunya, Digestive } \\
\text { Mego, Marianela; Hospital General Vall d'Hebron, Digestive System } \\
\text { Manichanh, chaysavanh; Hospital General Vall d'Hebron, } \\
\text { Canyellas, Nicolau; Universitat Rovira i Virgili } \\
\text { Cañueto, Daniel; Universitat Rovira i Virgili } \\
\text { Segurola, Hegoi; University Hospital Vall d'Hebron, Nutritional Support Unit } \\
\text { Jansana, Marta; University Hospital General of Catalonia } \\
\text { Malagelada, Carolina; Hospital General Vall d'Hebron, Digestive } \\
\text { Accarino, Anna; Hospital General Vall d'Hebron, } \\
\text { Vulevic, Jelena; University of Reading } \\
\text { Tzortziz, George; University of Reading } \\
\text { Gibson, Glenn; UNIVERSITY OF READING, FOOD MICROBIAL SCIENCES } \\
\text { UNIT (FMSU) } \\
\text { Saperas, Esteban; Hospital Universitari General de Catalunya } \\
\text { Guarner, Francisco; University Hospital Vall d'Hebron, Digestive System } \\
\text { Research Unit } \\
\text { Azpiroz, Fernando; HOSPITAL GENERAL VALL DHEBRON }\end{array}$ \\
\hline \hline Keywords: & \begin{tabular}{l} 
PREBIOTIC, DIET, GASTROINTESTINAL MOTILITY \\
\hline \hline
\end{tabular} \\
\hline
\end{tabular}




\section{EFFECT OF PREBIOTICS VERSUS LOW FODMAP DIET ON FUNCTIONAL GUT SYMPTOMS}

Short title: Prebiotics versus FODMAP diet

Jose-Walter Huaman ${ }^{1,2}$, Marianela Mego ${ }^{1}$, Chaysavanh Manichanh ${ }^{1}$, Nicolau Cañellas ${ }^{3,4}$, Daniel Cañueto ${ }^{3}$, Hegoi Segurola ${ }^{5}$, Marta Jansana ${ }^{6}$, Carolina Malagelada ${ }^{1}$, Anna Accarino ${ }^{1}$, Jelena Vulevic ${ }^{7}$, George Tzortzis ${ }^{7}$, Glenn Gibson $^{8}$, Esteban Saperas ${ }^{2}$, Francisco Guarner ${ }^{1}$, Fernando Azpiroz ${ }^{1}$.

1. Digestive System Research Unit, University Hospital Vall d'Hebron; Centro de Investigación Biomédica en Red de Enfermedades Hepáticas y Digestivas (Ciberehd); Universitat Autònoma de Barcelona, 08193 Bellaterra (Cerdanyola del Vallès), Spain.

2. Department of Gastroenterology, University Hospital General of Catalonia, San Cugat del Valles, Barcelona, Spain.

3. Metabolomics Platform, Universitat Rovira i Virgili, Campus Sescelades; Tarragona, Spain.

4. Centro de Investigación Biomédica en Red de Diabetes y Enfermedades Metabólicas (Ciberdem).

5. Nutritional Support Unit, University Hospital Vall d'Hebron, Barcelona, Spain.

6. Department of Endocrinology and Nutrition, University Hospital General of Catalonia, San Cugat del Valles, Barcelona, Spain.

7. Clasado Research Services Ltd, Science and Technology Centre, University of Reading, Early Gate, Reading, UK.

8. Food Microbial Sciences Unit, Department of Food and Nutritional Sciences, The University of Reading, Whiteknights, Reading, UK

Address for correspondence:
Fernando Azpiroz, M.D.

Digestive System Research Unit Hospital General Vall d'Hebron 08035-Barcelona, Spain

Phone: (34) 932746222

Fax: (34) 934894456

e-mail: azpiroz.fernando@gmail.com

Word count: 3941 


\begin{abstract}
Objective. To compare the effects of a prebiotic supplement versus a low FODMAP diet in patients with functional gut disorders. Design. Two-centre, parallel, randomized, doubleblind study comparing the effects of a prebiotic supplement $(2.8 \mathrm{~g} /$ day Bimuno, Clasado Biosciences, Jersey, Channel Islands) with a placebo diet (prebiotic group) versus and a placebo supplement (2.8 g per day xylose) with a low FODMAP diet (LFD group). The study consisted of 1 week of pretreatment (days 1-7), 4 weeks of treatment (days 8-35) and 2 weeks of post-treatment follow-up (days 36-49). All patients received dietary instructions in similar intervention visits. The following outcomes were measured: a) the number of daytime gas evacuations over the course of 2 days were measured by means of an event marker; b) the volume of gas evacuated over the course of 4 hours after consumption of a probe meal were measured via a rectal tube; and c) the microbiota composition was measured by faecal Illumina MiSeq sequencing.
\end{abstract}

Results. Both treatments improved the symptoms to a similar extent. After termination of the prebiotic treatment, the symptomatic improvement persisted for 2 weeks. In contrast, after termination of the LFD diet, a rebound was observed, and by 2 weeks post-treatment, the symptoms were more severe than during the treatment phase. In the prebiotic group, the counts of bifidobacteria increased, and the counts of sulphate-producing species decreased, while the opposite effects were observed in the LFD group.

Conclusion. Both the LFD and the B-GOS prebiotic administration were similarly effective at treating gas-related symptoms in patients with functional gut disorders. However, both strategies had different consequences after treatment discontinuation; while the 
improvement of symptoms persisted 2 weeks after prebiotic administration was discontinued, the symptoms relapsed after the termination of the LFD.

Keywords: intestinal gas, microbiota, prebiotics, low FODMAP diet, functional gastrointestinal symptoms 


\section{Summary 'box'}

1. What is already known about this subject?

- Diets low in fermentable residues, such as low FODMAP diet, improve gas-related symptoms in patients with functional gut disorders.

- Paradoxically, a galactooligosaccharide prebiotic, B-GOS, that is not absorbed in the small bowel and is selectively fermented by colonic microbiota, produces a similar effect on gas-related symptoms.

2. What are the new findings?

- Both a low FODMAP diet (plus a placebo supplement; $2.8 \mathrm{~g}$ per day xylose) and a prebiotic supplement (2.8 g/day Bimuno, Clasado Biosciences, Jersey, Channel Islands; plus a placebo diet) were similarly effective at treating gas-related symptoms in patients with functional gut disorders.

- However, both strategies had different consequences after treatment discontinuation; while the improvement of symptoms persisted 2 weeks after prebiotic administration was discontinued, the symptoms relapsed after the termination of the

3. How might it impact on clinical practice in the foreseeable future?

- Since dietary restrictions, particularly the low FODMAP diet, are cumbersome to follow, these results present an alternative patient management strategy.

- Furthermore, given the sustained effect of B-GOS compared to the reversible effects of the low FODMAP diet, intermittent treatment with B-GOS might represent an additional advantage over the continuous treatment required with the low 


\section{FODMAP diet.}




\section{INTRODUCTION}

A significant proportion of patients with functional gut disorders complain of symptoms that they attribute to intestinal gas, such as flatulence, abdominal bloating, distension and borborygmi ${ }^{1}$. A series of studies has shown that diets low in fermentable residues, i.e., those that contain low amounts of products that escape small bowel absorption and are fermented by colonic microbiota, improve gas-related symptoms in these patients. Similar effects have been observed with different diets ${ }^{2-5}$, among which a diet low in fermentable oligo-, di-, and monosaccharides and polyols (FODMAPs) has become particularly popular and widely used ${ }^{46}$. Paradoxically, a galactooligosaccharide prebiotic, B-GOS, that is not absorbed in the small bowel and is selectively fermented by colonic microbiota, produces a similar effect on gas-related symptoms ${ }^{7}$. Initially, this product increases the fermentive activity of microbiota and, consequently, increases gas production, but this effect declines after a relatively short adaptation period (1-2 weeks) with a shift in microbiota metabolism towards more efficient, low-gas producing pathways ${ }^{8}$ 9. In contrast, some data have indicated that in the long run, a low FODMAP diet with restricted fermentable substrates may negatively affect gut microbiota ${ }^{10-12}$.

Based on these data, we hypothesized that both approaches, i.e., a low FODMAP diet and a B-GOS prebiotic supplement, would improve gas-related symptoms during the period of administration but would have different consequences after discontinuation, due to their specific effects on gut microbiota. Hence, our aim was to compare the effects of a low FODMAP diet versus the B-GOS prebiotic supplement on gas-related symptoms and colonic microbiota during a 4-week treatment period and a 2-week post-treatment period. 
To this aim, consecutive patients complaining of excessive anal gas evacuation, i.e., flatulence, were recruited. We compared the effects of a low FODMAP diet plus a placebo supplement versus a placebo diet (Mediterranean-type) plus the B-GOS prebiotic supplement on gas-related symptoms and anal gas evacuation. The effects of the treatments were evaluated during basal conditions, after a 3-day challenge diet and after a probe meal.

\section{MATERIAL AND METHODS}

\section{Study design}

This study was a randomized, two-centre, parallel and double-blind study performed between August 2014 and July 2015 in 2 tertiary care referral centres. The study protocol was registered with ClinicalTrials.gov (NCT02210572). All co-authors had access to the study data and reviewed and approved the final manuscript. The study compared the effect of a prebiotic supplement plus a placebo diet (prebiotic group) versus a placebo supplement plus a low FODMAP diet (LFD group; see sections on supplement products and treatment diets below). The study lasted 7 weeks (49 days) with 3 phases: a 1-wk pretreatment phase (days 1-7), a 4-wk treatment phase (days 8-35) and 2-wk posttreatment phase (days 36-49) (Figure 1).

The primary outcome was the effect of the treatments on gut microbiota composition, specifically the relative abundance of bifidobacteria. Secondary outcomes were intestinal gas production, as an index of microbiota activity, and digestive sensations. 


\begin{abstract}
Randomization and masking
Participants were randomized using a block design ( $n=10$ per block) by a computergenerated randomization list. Participants did not know which treatment they were assigned, and all patients received dietary instructions following the same schema of intervention visits. The investigators performing the tests and the clinical follow-up were blinded to the intervention (supplement and diet).
\end{abstract}

\title{
Participants
}

Patients fulfilling the criteria of having a functional gastrointestinal disorder and complaining of excessive anal gas evacuation (i.e., flatulence) ( 35 women, 5 men; age range $24-73$ years) participated in the study. Antibiotic consumption during the previous 2 months was an exclusion criterion. Participants were instructed to fill out a clinical questionnaire to evaluate bowel habits and gastrointestinal symptoms. Subjects gave written informed consent to participate in the study. The protocol for the study had previously been approved by the Institutional Review Board of University Hospital Vall d'Hebron.

\section{Supplement product}

During the treatment phase (days 8-35) participants consumed 1 sachet per day of either prebiotic (2.8 g per day Bimuno containing 1.37g B-GOS, Clasado Biosciences , Jersey, Channel Islands) or placebo (2.8 g xylose). 


\section{Dietary instructions}

The treatment diet, either a low FODMAP or a placebo diet, was administered during the first 23 days of the treatment phase (days 8-31). Participants were put on a standard, highly flatulogenic diet during 3-day periods at the end of the pretreatment phase (days 5-7) and the treatment phase (days 33-35). During the first 4 days of the pretreatment phase (days 1-4) and the post-treatment phase (days 36-49), the participants consumed their habitual diet. For the duration of the study, patients were not allowed to consume any fermented dairy products or any tablets, pills or food supplements containing pre- or probiotics other than those provided. A dietician provided dietary instructions and checked adherence to the diets using structured consumption questionnaires.

\section{$\underline{\text { Treatment diets }}$}

Low FODMAP diet. The food list for the diet was based on published information adapted to local eating habits ${ }^{13-15}$. The diet specifically excluded a) dairy products, b) fruit, including apples, pears, peaches, apricots, cherries, mangoes, watermelon, melon, and prunes, c) legumes, including chickpeas, white beans, lentils, and soya beans, d) vegetables, including artichokes, asparagus, beetroot, Brussels sprouts, broccoli, cabbage, garlic, leeks, onions, peas, lettuce, and cauliflower, e) cereals, including bread, rye, and oats, and f) sweeteners.

Placebo diet. The diet was balanced and Mediterranean-type ${ }^{2}$, including the following every day: a) meat, fowl, fish, or eggs, b) vegetables, salad or legumes, c) bread, rice, pasta, potatoes or cereals, d) dairy products, and e) fruits. 


\section{Challenge diet}

The challenge diet consisted of: a) breakfast of wholemeal cookies (39 g) plus coffee, tea and/or milk, b) lunch of white beans (200 g), mixed vegetables (250 g) or chickpeas (200 g) and wholemeal bread (50 g), plus meat, fowl or fish and fruit (banana, figs peaches or prunes), and c) dinner of vegetable soup (200 mL), wholemeal bread (50 g) and fruit (banana, figs, peaches or prunes).

\section{Outcomes}

The outcomes were measured during 3-day periods at 5 time points throughout the study: in the pretreatment phase just before and during the challenge diet (days 2-4 and 57, respectively), in the treatment phase just before and during the challenge diet (days 3032 and 33-35, respectively), and at the end of the post-treatment phase (days 47-49) (Figure 1).

\section{Daily symptom questionnaire}

During the 3 days of each evaluation period, the participants were instructed to fill out daily questionnaires that included the following parameters: (a) subjective sensations of flatulence (defined as anal gas evacuation), abdominal bloating (pressure/fullness), abdominal distension (sensation of girth increase), borborygmi and abdominal discomfort/pain using 0-10 analogue scales, (b) digestive well-being using a 10-point scale graded from +5 (extremely pleasant sensation/satisfaction) to -5 (extremely unpleasant 
sensation/dissatisfaction), and (c) mood on similar scale graded from +5 (very positive) to -5 (very negative). For each symptom, the scores for each 3-day period were averaged. This questionnaire has been previously used and has been shown to be sensitive enough to detect the effects of dietary interventions in different populations ${ }^{216-18}$.

\section{Number of anal gas evacuations}

The number of anal gas evacuations during the last 2 days of each evaluation period were measured and averaged. Participants were instructed to carry an event marker (Hand Tally Counter No 101, Digi Sport Instruments, Shanggiu, China) during the day and to use it to register each passage of anal gas. This method has been previously used with reproducible and consistent results ${ }^{28916}$; furthermore, studies measuring the number of gas evacuations by an event marker and continuously recording anal gas evacuations have shown a very good correlation between the results of the two methods $(R>0.95 ; p<0.05)^{19-}$ 22.

\section{$\underline{\text { Response to a probe meal }}$}

The test was performed the day after the pretreatment and treatment phases. Participants reported to the laboratory after an overnight fast and consumed a probe meal. The probe meal consisted of a ham omelette (1 egg, $30 \mathrm{~g}$ sliced ham cooked with $5 \mathrm{~g}$ oil), $46 \mathrm{~g}$ of white bread, $10 \mathrm{~g}$ of butter, $25 \mathrm{~g}$ of jam and $200 \mathrm{~mL}$ of fruit juice $(400 \mathrm{Kcal}, 350 \mathrm{~mL}$ total volume, $1.5 \mathrm{~g}$ of fibre).

The volume of gas evacuated by anus was measured for $4 \mathrm{~h}$ after the probe meal, as previously described ${ }^{162324}$. Briefly, gas was collected using a rectal balloon catheter (20 
F Foley catheter, Bard, Barcelona, Spain) connected via a gas-tight line to a barostat, and the volume was continuously recorded. The intrarectal balloon was inflated with $5 \mathrm{~mL}$ of water to prevent anal gas leaks.

Patients' perceptions of abdominal sensations were measured every 30 min during the 4-h gas collection period using the same scales as described above: 0 to 10 scales for scoring abdominal bloating (pressure/fullness), abdominal distension (sensation of girth increase), borborygmi and abdominal discomfort/pain and -5 to +5 scales for scoring digestive well-being and mood.

\section{Metabolomic analysis}

Urine was collected for $24 \mathrm{~h}$ during the last day of the pretreatment and treatment periods (days 7 and 35, respectively) from 22 patients, with 11 from each treatment group. For each sample, a one-dimensional $1 \mathrm{H}$ NMR spectrum was acquired according to the standard recommendations ${ }^{2526}$. Analyses of the histamine levels and P-cresol level in the urine samples were performed by mass spectroscopy.

\section{Microbiota composition}

Faecal samples were collected from 40 patients during the pretreatment, treatment and post-treatment phases (days 5, 32 and 49, respectively) (Figure 1). After collection and homogenization, the samples were immediately frozen by the participants in their home freezers at $-20^{\circ} \mathrm{C}$ and later brought to the laboratory in a freezer pack, where they were stored at $-80^{\circ} \mathrm{C}$. Microbiota analysis was performed as previously described (see 
Supplemental material).

\section{Statistical analysis}

Sample size calculation. Sample size calculation was performed based on the effect of the prebiotic B-GOS on fecal bifidobacteria. In previous studies ${ }^{8}$, B-GOS cosumption increased relative abundande of bifidobateria in 13 out 20 subjects (65\%); by contrast, low FODMAP diet did not induce this effect ${ }^{12}$. Assuming a bifidobacteria increase in $65 \%$ of patients on B-GOS and in less than $15 \%$ of patients on low FODMAP diet, it was estimated that a sample size of 18 individuals per group would provide a $90 \%$ power to detect statistical differences between groups.

Metabolomic analysis. Differences from pre- and post-treatment spectra were used for comparisons between groups.

Microbiota analysis. The Shapiro-Wilk test was used to assess the normality of the data, and pairwise comparisons were made between the study groups with the nonparametric Kruskal-Wallis one-way analysis of variance test, which compares means between groups. A false discovery rate (FDR) of corrected $p$-values was taken into account when considering the significance of the results.

Overall comparisons. In each group, the effect of treatment (treatment minus pretreatment) was measured at the baseline, after the challenge diet and after the probe meal. The effects of treatment were compared between the prebiotic and the LFD group. The means $( \pm S E)$ of the measured variables were calculated. The Kolmogorov-Smirnov 


\begin{abstract}
test was used to check the normality of the data distribution. Parametric normally distributed data were compared by Student's $t$-test for paired or unpaired data; otherwise, the Wilcoxon signed-rank test was used for paired data, and the Mann-Whitney $U$ test was used for unpaired data. The association of parameters was analysed using linear regression.
\end{abstract}

RESULTS

Demographics

Forty-four patients were selected and included in the study (Figure 2 and Table 1). All patients $(n=44)$ had a functional disorder diagnosis based on the Rome III criteria 2728 (Table 1). No demographic or clinical differences were found between the prebiotic and LFD groups (Table 1).

\title{
Symptoms and gas evacuation
}

Pre-treatment phase. On their habitual diets, the patients exhibited mild to moderate symptoms (Figure 3) and recorded 15 \pm 1 evacuations of gas during the daytime (Figure 4). No significant differences in the above parameters were detected between the study groups (Figures 3, 4).

Treatment phase. Both treatments improved the symptom scores in the daily questionnaires (Figure 3). The LFD diet, but not the prebiotic treatment, significantly reduced the number of gas evacuations (Figure 4). No differences in the effect of treatment (treatment values minus pretreatment values) were detected between the study groups 
$(p=0.287$ by MANOVA $)$.

Post-treatment phase. With the prebiotic treatment, the symptomatic improvement was maintained 2 weeks after treatment termination; to note, digestive well-being further improved. In contrast, after the LFD diet was terminated, a rebound was observed, and by 2 weeks post-termination, the symptoms were greater than during treatment phase (Figure 3 ), although the change (from treatment values to post-treatment values) was not significantly different between groups ( $p=0.091$ by MANOVA). In the prebiotic group, the number of gas evacuations after treatment was slightly (not significantly) lower than in the pretreatment and the treatment phases. In contrast, in the LFD group, the number of gas evacuations after treatment increased compared to the treatment level $(p=0.056$ vs treatment; $p=0.059$ vs change in prebiotic group) (Figure 4).

\section{Response to challenge diet}

Pre-treatment phase. The challenge diet increased the intensity of symptoms similarly in both groups (Figure 5). The number of daytime gas evacuations increased in the prebiotic and the LFD groups (by $12 \pm 4$ evacuations and $6 \pm 2$ evacuations, respectively; $p=0.160$ between groups; $p \leq 0.005$ vs habitual diet for both).

Treatment phase. The effect of the challenge diet on symptoms (changes in daily symptoms) during treatment was similar that observed before treatment in both groups and the effect was not different between treatments ( $p=0.148$ by MANOVA) (Figure 5$)$. Since the symptoms during both treatments were at lower levels than in the pretreatment phase, the symptoms due to the challenge diet were lower during in the treatment phase than 
during the pretreatment phase in both groups. The increase in the number of gas evacuations in response to the challenge diet was similar to the increase observed during the pretreatment phase in both groups (increase of $12 \pm 3$ evacuations in the prebiotic group and $7 \pm 2$ evacuations in the LFD group; $p=0.117$ between groups; $p \leq 0.002$ vs habitual diet for both).

\section{Response to probe meal}

Pre-treatment phase. After the probe meal, participants reported symptoms (Figure 6), and both the symptom scores as well as the total volume of gas evacuated by anus in the 4-hour postprandial period $(149 \pm 20 \mathrm{~mL}$ in the prebiotic group and $173 \pm 22 \mathrm{~mL}$ in the LFD group) were similar in both groups (Figure 6).

Treatment phase. In both groups, the probe meal produced the same volume of gas in the treatment phase $(140 \pm 17 \mathrm{~mL}$ in prebiotic and $160 \pm 29 \mathrm{~mL}$ in LFD group) and in the pretreatment phase, but overall, the tolerance of the probe meal was somewhat better during the treatment phase in both groups (Figure 6), without differences between groups $(p=0.570$ by MANOVA $)$.

\section{Metabolomic analysis}

The initial analysis was performed using a fingerprinting approach. Significant differences between both treatments were detected in five integrated regions of the spectrum. These regions were located at 7.911-7.898 ppm, 4.165-4.148 ppm, 2.789-2.786 ppm, 2.003-1.956 ppm, and 1.677-1.662 ppm. Using the Human Metabolome Database 
(HMDB) ${ }^{29}$ and STOCSY ${ }^{30}$, only two of these regions were identified as previously described metabolites, xanthine and an unsaturated fatty acid. A partial least squares discriminant analysis (PLS-DA) classifi using the five integrated regions that were different between groups showed good discrimination between treatments: All patients were correctly classified according to their treatment. In addition to the integrated regions, the metabolite 4-deoxythreonic acid, a metabolite previously found to decrease in urine incubated with $E$. coli, was found to have significant weight in the model ${ }^{31}$. No significant differences were found in the concentrations of histamine or P-cresol in urine samples according to treatment or group.

\section{Changes in faecal microbiota}

In the LFD group, treatment was associated with a decrease in the relative abundance of unknown Clostridiales and Bacteroides species and an increase in the abundance of species belonging to Ruminococcaceae (mucin degraders), Desulphovibronaceae (sulphate-reducing bacteria) and Enterobacteriaceae families (Table 2). In the post-treatment phase, changes in several bacterial taxa were detected, including a remarkable increase in the abundance of members of the Bifidobacterium genus (Table 2). In the prebiotic group, the abundance of two Lachnospiraceae species increased during treatment (Table 3). There was a reduction in the abundance of Parabacteroides, Oscillospira, Barnesiellaceae, Christensenellaceae, and Bilophila wadsworthia species. Figure 7 shows trade-off in response to the interventions between the abundance of Bilophila wadsworthia and that of bifidobacteria. In the LFD group, the counts of 
bifidobacteria went down during treatment, but rebounded in the post-treatment phase, whereas in the prebiotic group, they showed a positive change during treatment and no change after treatment. In contrast, the change in the counts of Bilophila wadsworthia during treatment was positive in the LFD group and negative in the prebiotic group, and the trend persisted in the post-treatment phase. Interestingly, in the LFD group, the increase in gas frequency in the post-treatment phase correlated with the increase in Bilophila wadsworthia abundance $(r=0.48, p=0.050)$, and this correlation was absent in the prebiotic group $(r=0.07, p=0.796)$.

\section{DISCUSSION}

Our study shows that both the LFD and the B-GOS prebiotic supplement are similarly effective in the treatment of gas-related symptoms in patients with functional gut disorders. However, both strategies had different consequences after treatment discontinuation. While the improvement of symptoms persisted 2 weeks after prebiotic administration, symptoms relapsed after discontinuing the LFD.

Most likely, the improvement in symptoms in response to each treatment is achieved via different mechanisms. The clinical outcome associated with the LFD in our study is similar to that previously reported ${ }^{4611}$. The LFD reduced gas production, and conceivably this effect contributed to the improvement of gas-related symptoms. Diets low in residues are associated with lower volumes of colonic content ${ }^{32}$, and conceivably this is also the case with the LFD. Intestinal loads of FODMAPs have been shown to increase the volume of content within the small bowel ${ }^{33}$, but whether the intestinal content on an LFD is lower 
than on a normal diet without an excess of FODMAPs remains unproven.

The improvement of gas-related symptoms due to supplementation with the B-GOS prebiotic in our study is consistent with previous observations ${ }^{7}$. However, the mechanisms by which B-GOS improves symptoms are not clear. As described above, B-GOS is a nonabsorbable, fermentable product; consequently, it serves as substrate for colonic microbiota and at initial administration increases intestinal gas production. However, continuous B-GOS administration elicits an adaptation phenomenon, resulting in a progressive decrease in gas production back to pre-administration levels by $7-10$ days of treatment ${ }^{89}$ and a change in gut microbiota profile ${ }^{78}$, which is likely the cause of adaptation. Furthermore, B-GOS has shown anti-inflammatory effects, and it could also reduce intestinal hypersensitivity, a characteristic feature in patients with functional gut disorders that leads to symptoms in response to normal gut contents ${ }^{34} 35$. It is not known whether the effects of B-GOS administration on symptoms and microbiota are productspecific or are common to other prebiotics.

Symptom improvement persisted 2 weeks after termination of B-GOS administration. This post-administration effect is probably related to the changes in microbiota, and the current data do not indicate for how long it may persist. In contrast to the post-treatment effect of B-GOS, symptoms relapsed 2 weeks after LFD discontinuation, reflecting a cessation of the influence of LFD on the intraluminal environment when replaced by the patients' habitual diets.

Potentially beneficial effects of B-GOS on microbiota include the increase in Lachnospiraceae, which produce butyrate and other short chain fatty acids, and the 
decrease in Bilophila wadsworthia, a sulphate-reducing bacterium that has been found to play a role in individuals complaining of excess flatus ${ }^{16}$ and has been associated with intestinal inflammation in experimental models ${ }^{36}$. The changes in microbiota induced by LFD were distinctively different than those induced by B-GOS, particularly in relation to the abundance of bifidobacteria (decreased with LFD and increased with B-GOS) and Bilophila wadsworthia (increased with LFD and decreased with B-GOS).

As in previous studies in patients with functional gut disorders ${ }^{16}$, the challenge diet in the pretreatment period substantially increased anal gas evacuations and symptom scores, and a similar increase was observed in the pretreatment and treatment periods. However, since the patients felt better with the treatments, the challenge was better tolerated in terms of absolute symptom scores. In contrast to what we anticipated, tolerance of the challenge diet was similar with both treatments; conceivably, the challenge was too strong and overcame the potential influences of the treatments.

Likewise, the probe meal was better tolerated, i.e., with lower postprandial symptom scores, during both treatments than before both treatments, but without differences between groups. Furthermore, the treatments did not affect the volume of gas produced after the probe meal. This unexpected lack of differences could be because the probe meal was administered after 3 days on the challenge diet, which provided a heavy colonic load and blurred the effect of treatment. Indeed, intestinal gas production depends not only on recent but also on previous fermentable residue loads ${ }^{37}$.

The treatments produced different changes in urine metabolites. Although the significance of the changes is difficult to ascertain, the metabolomic analysis provided a 
good discrimination between groups, which served as an indirect index of the adherence to the dietary instructions. Previous studies have shown that a low FODMAP diet modulates histamine levels in urine ${ }^{38}$ and that $\mathrm{P}$-cresol, a bacterial protein fermentation metabolite, reflects the influence of diet on amino acid fermentation in the colon ${ }^{39}$; however, no changes in these metabolites were detected in our study.

From a practical perspective, our study indicates that in the short run, daily administration of B-GOS prebiotic is equally effective as an LFD. Since dietary restrictions, particularly the LFD, are cumbersome to follow, these results present an alternative patient management strategy. Furthermore, given the sustained effect of B-GOS compared to the reversible effects of LFD, intermittent treatment with B-GOS might represent an additional advantage over the continuous treatment required with LFD. Recent data indicate that simultaneous administration of a probiotic may prevent the potentially deleterious influences of LFD on microbiota ${ }^{11}$. Possibly, the combination of different therapies may have synergistic effects. Gas-related symptoms may benefit from individualized treatment with diet, prebiotics and/or probiotics in single or combined therapy. Ideally, individual treatment strategies would be based on biological markers, including microbiota metabolic activity and composition. 


\section{Statements}

Acknowledgments. The authors thank Gloria Santaliestra for secretarial assistance.

Writing Assistance. American Journal Experts for English editing of the manuscript (Certificate Verification Key; 2B02-9D8E-A5F0-B802-AAAC) funded by SAF 2016-76648-R

Grants. The present study was supported by the Spanish Ministry of Economy and Competitiveness (Dirección General de Investigación Científica y Técnica, SAF 201676648-R); Ciberehd is funded by the Instituto de Salud Carlos III.

\section{Authorship Statement: \\ Guarantor of the article: Fernando Azpiroz}

\section{Author contributions:}

JWH: study management, conduction of experiments and data analysis.

MM: study management, conduction of experiments and data analysis.

C Manichanh: microbiota analysis

DC: metabolomic analisis

NC: metabolomic analisis

HS: dietary guidance

MJ: dietary guidance

C Malagelada: metabolomic analysis 
AA: supervision of studies.

JV: study design, interpretation of results, manuscript revision

GT: study design, interpretation of results

GG: study design, interpretation of results, manuscript revision

ES: study design, manuscript revision

FG: study design, data interpretation manuscript revision

FA. study design, and manuscript preparation.

All authors approved the final draft of the manuscript.

\section{Competing interests}

Jelena Vulevic, employee Clasado.

George Tzortzis, employee Clasado.

Glen Gibson, Francisco Guarner and Fernando Azpiroz served as advisory board members for Clasado.

Rest of authors, no competing interests declared. 


\section{References}

1. Azpiroz F, Guyonnet D, Donazzolo Y, et al. Digestive Symptoms in Healthy People and Subjects With Irritable Bowel Syndrome: Validation of Symptom Frequency Questionnaire. J Clin Gastroenterol 2014

2. Azpiroz F, Hernandez C, Guyonnet D, et al. Effect of a low-flatulogenic diet in patients with flatulence and functional digestive symptoms. Neurogastroenterol Motil 2014;26(6):779-85.

3. Bohn L, Storsrud S, Liljebo T, et al. Diet Low in FODMAPs Reduces Symptoms of Irritable Bowel Syndrome as Well as Traditional Dietary Advice: A Randomized Controlled Trial. Gastroenterology 2015;149(6):1399-407.

4. Halmos EP, Power VA, Shepherd SJ, et al. A Diet Low in FODMAPs Reduces Symptoms of Irritable Bowel Syndrome. Gastroenterology 2014;146(1):67-75.

5. Eswaran SL, Chey WD, Han-Markey T, et al. A Randomized Controlled Trial Comparing the Low FODMAP Diet vs. Modified NICE Guidelines in US Adults with IBS-D. Am J Gastroenterol 2016;111(12):1824-32. doi: 10.1038/ajg.2016.434

6. Krogsgaard LR, Lyngesen M, Bytzer P. Systematic review: quality of trials on the symptomatic effects of the low FODMAP diet for irritable bowel syndrome. Aliment Pharmacol Ther 2017;45(12):1506-13. doi: 10.1111/apt.14065

7. Silk DB, Davis A, Vulevic J, et al. Clinical trial: the effects of a transgalactooligosaccharide prebiotic on faecal microbiota and symptoms in irritable bowel syndrome. AlimentPharmacolTher 2009;29(5):508-18.

8. Mego M, Manichanh C, Accarino A, et al. Metabolic adaptation of colonic microbiota to 
galactooligosaccharides: a proof-of-concept-study. Aliment Pharmacol Ther 2017;45(6):670-80.

9. Mego M, Accarino A, Tzortzis G, et al. Colonic gas homeostasis: Mechanisms of adaptation following HOST-G904 galactooligosaccharide use in humans. Neurogastroenterol Motil 2017;29(9) doi: 10.1111/nmo.13080

10. Staudacher HM, Lomer MC, Anderson JL, et al. Fermentable carbohydrate restriction reduces luminal bifidobacteria and gastrointestinal symptoms in patients with irritable bowel syndrome. J Nutr 2012;142(8):1510-18.

11. Staudacher HM, Lomer MCE, Farquharson FM, et al. A Diet Low in FODMAPs Reduces Symptoms in Patients With Irritable Bowel Syndrome and A Probiotic Restores Bifidobacterium Species: A Randomized Controlled Trial. Gastroenterology 2017;153(4):936-47. doi: 10.1053/j.gastro.2017.06.010

12. Halmos EP, Christophersen CT, Bird AR, et al. Diets that differ in their FODMAP content alter the colonic luminal microenvironment. Gut 2015;64(1):93-100.

13. Muir JG, Rose R, Rosella O, et al. Measurement of short-chain carbohydrates in common Australian vegetables and fruits by high-performance liquid chromatography (HPLC). J AgricFood Chem 2009;57(2):554-65.

14. Biesiekierski JR, Rosella O, Rose R, et al. Quantification of fructans, galactooligosacharides and other short-chain carbohydrates in processed grains and cereals. JHumNutrDiet 2011;24(2):154-76.

15. Odimet, Organizador dietético Metabólico. Hospital Clínico Universitario de Santiago de Compostela, Spain 2014 
16. Manichanh C, Eck A, Varela E, et al. Anal gas evacuation and colonic microbiota in patients with flatulence: effect of diet. Gut 2014;63:401-08.

17. Malagelada C, Barba I, Accarino A, et al. Cognitive and hedonic responses to meal ingestion correlate with changes in circulating metabolites. Neurogastroenterol Mot $2016 ; 28(12): 1806-14$.

18. Malagelada C, Accarino A, Molne L, et al. Digestive, cognitive and hedonic responses to a meal. Neurogastroenterol Motil 2015;27(3):389-96.

19. SerraJ, Azpiroz F, Malagelada JR. Gastric distension and duodenal lipid infusion modulate intestinal gas transit and tolerance in humans. Am J Gastroenterol 2002;97:2225-30.

20. Serra J, Azpiroz F, Malagelada JR. Mechanisms of intestinal gas retention in humans:impaired propulsion versus obstructed evacuation. AmJPhysiol 2001;281:G138-G43.

21. Serra J, Azpiroz F, Malagelada JR. Intestinal gas dynamics and tolerance in humans. Gastroenterology 1998;115:542-50.

22. Serra J, Azpiroz F, Malagelada JR. Impaired transit and tolerance of intestinal gas in the irritable bowel syndrome. Gut 2001;48:14-19.

23. Serra J, Salvioli B, Azpiroz F, et al. Lipid-induced intestinal gas retention in the irritable bowel syndrome. Gastroenterology 2002;123:700-06.

24. Hernando-Harder AC, Serra J, Azpiroz F, et al. Colonic responses to gas loads in subgroups of patients with abdominal bloating. Am J Gastroenterol 2010;105:87682. 
25. Emwas AH, Roy R, McKay RT, et al. Recommendations and Standardization of Biomarker Quantification Using NMR-Based Metabolomics with Particular Focus on Urinary Analysis. J Proteome Res 2016;15(2):360-73. doi: 10.1021/acs.jproteome.5b00885

26. Emwas $\mathrm{AH}$, Luchinat $\mathrm{C}$, Turano $\mathrm{P}$, et al. Standardizing the experimental conditions for using urine in NMR-based metabolomic studies with a particular focus on diagnostic studies: a review. Metabolomics 2015;11(4):872-94. doi: 10.1007/s11306-014-07467

27. Stanghellini V, Chan FK, Hasler WL, et al. Gastroduodenal Disorders. Gastroenterology 2016;150(6):1380-92.

28. Lacy BE, Mearin F, Chang L, et al. Bowel Disorders. Gastroenterology 2016;150(6):1393-407. doi: 10.1053/j.gastro.2016.02.031

29. Wishart DS. Human Metabolome Database: completing the 'human parts list'. Pharmacogenomics 2007;8(7):683-6. doi: 10.2217/14622416.8.7.683

30. Cloarec O, Dumas ME, Trygg J, et al. Evaluation of the orthogonal projection on latent structure model limitations caused by chemical shift variability and improved visualization of biomarker changes in $1 \mathrm{H}$ NMR spectroscopic metabonomic studies. Anal Chem 2005;77(2):517-26. doi: 10.1021/ac048803i

31. Maeda K, Shiraishi S, Sakamoto N, et al. Identification of Escherichia coli by detection of hydroquinone and uracil in the urine system. J Chromatogr 1985;345(1):11-8.

32. Bendezu RA, Mego M, Monclus E, et al. Colonic content: effect of diet, meals, and defecation. Neurogastroenterol Motil 2016;Feb 12. doi: 10.1111/nmo.12930. [Epub 
ahead of print]. PMID: 27545449

33. Murray K, Wilkinson-Smith V, Hoad C, et al. Differential effects of FODMAPs (fermentable oligo-, di-, mono-saccharides and polyols) on small and large intestinal contents in healthy subjects shown by MRI. Am J Gastroenterol 2014;109(1):11019.

34. Major G, Pritchard S, Murray K, et al. Colon hypersensitivity to distension, rather than excessive gas production, produces carbohydrate-related symptoms in individuals with irritable bowel syndrome. Gastroenterology 2017;152(1):124-33.

35. Boeckxstaens G, Camilleri M, Sifrim D, et al. Fundamentals of Neurogastroenterology: Physiology/Motility - Sensation. Gastroenterology 2016;150:1292-304.

36. Devkota S, Wang Y, Musch MW, et al. Dietary-fat-induced taurocholic acid promotes pathobiont expansion and colitis in II10-/- mice. Nature 2012;487(7405):104-8. doi: $10.1038 /$ nature 11225

37. Mego M, Accarino A, Malagelada JR, et al. Accumulative effect of food residues on intestinal gas production. Neurogastroenterol Motil 2015;27(11):1621-28.

38. Mclntosh K, Reed DE, Schneider T, et al. FODMAPs alter symptoms and the metabolome of patients with IBS: a randomised controlled trial. Gut 2017;66(7):1241-51. doi: 10.1136/gutjnl-2015-311339

39. de Preter V, Vanhoutte T, Huys G, et al. Baseline microbiota activity and initial bifidobacteria counts influence responses to prebiotic dosing in healthy subjects. Aliment Pharmacol Ther 2008;27(6):504-13. doi: 10.1111/j.1365-2036.2007.03588.x 


\title{
FIGURE LEGENDS
}

\author{
Figure 1. Experimental design. Note the color code: pre-treatment phase white, \\ treatment black and post-treatment grey.
}

\section{Figure 2. Flow chart.}

Figure 3. Baseline symptoms measured by daily questionnaires pre-treatment (days 24 , white), in the treatment phase (days $30-32$, black) and in the posttreatment phase (day 47-49, grey) (LFD $n=21$; prebiotic group $n=19)$. Data are average over each 3-day periods.
Figure 4. Number of daytime anal gas evacuations during the last 2 days of each evaluation phase: pre-treatment (Pre: days 3-4, white), treatment (Tx: days 31-32, black) and post-treatment (Post: days 48-49, grey) (LFD $n=21$; prebiotic group $n=19$ ).
Figure 5. Effect of challenge diet on symptoms measured by daily questionnaires pre- treatment (days 5-7, white) and in the treatment phase (days 33-35, black) (LFD $n=21$; prebiotic group $n=19$ ). Data are average over each 3-day periods. In each group the change during treatment from pre-treatment phase is shown.


Figure 6. Symptoms in response to probe meal in the pre-treatment (day 5, white) and in the treatment phase (day 33, black) (LFD $n=21$; prebiotic group $n=19$ ).

Figure 7. Effect of treatment on relative abundance in Bifidobacterium and Bilophila wadsworthia. Figure shows changes during treatment (from pre-treatment phase) and after treatment (from treatment phase). Overall differences were tested by non-parametric Kruskal-Wallis analysis of variance on ranks, and the Mann-Whitney test was used for post-hoc comparisons (LFD $n=21$; prebiotic group $n=19)$. Data are median and interquartile range. 
Table 1. Demographics and clinical data

\begin{tabular}{llll} 
& \multicolumn{3}{c}{ STUDY GROUPS } \\
\cline { 2 - 4 } - Age (range), years & $43(24-73)$ & $48(26-69)$ & 0.309 \\
\cline { 2 - 4 } - Sex, M/F & $2 / 17$ & $3 / 18$ & 1 \\
- Diagnosis, IBS-a/IBS-d/FAP & $9 / 5 / 5$ & $9 / 5 / 7$ & 0.889 \\
- Bowel habit, No./wk & $6.1 \pm 0.8$ & $5.9 \pm 0.7$ & 0.679 \\
- Stool form, Bristol score & $4.9 \pm 1.0$ & $4.8 \pm 1.0$ & 0.797 \\
- Symptom duration, years & $4.7 \pm 1.8$ & $4.4 \pm 1.5$ & 0.694 \\
- Flatulence, score* & $5.2 \pm 3.0$ & $5.3 \pm 2.3$ & 0.976 \\
- Abdominal bloating* & $5.4 \pm 2.4$ & $5.9 \pm 2.2$ & 0.474 \\
- Abdominal distension* & $5.7 \pm 2.2$ & $6.3 \pm 2.2$ & 0.374 \\
- Borborigmi* & $3.1 \pm 2.3$ & $3.8 \pm 2.5$ & 0.348 \\
- Discomfort/pain, score* & $4.7 \pm 1.8$ & $4.8 \pm 2.9$ & 0.975
\end{tabular}

FAP, functional abdominal pain; * average of daily measurements over 3-day pre-treatment evaluation period 
Table 2. Changes in bacterial taxa during and after intervention in the LFD group

\begin{tabular}{|c|c|c|}
\hline \multicolumn{3}{|c|}{ Changes during treatment from pre-treatment phase } \\
\hline TAXA & $p^{1}$ & Trend \\
\hline Clostridiales; unknown genus; species 324 & $<0.01$ & $\downarrow$ \\
\hline Lachnospiraceae; unknown genus; species 40 & $<0.02$ & 个 \\
\hline Ruminococcaceae; unknown genus; species 53 & $<0.03$ & $\uparrow$ \\
\hline Bacteroidales; unknown genus; species 39 & $<0.03$ & 个 \\
\hline Ruminococcaceae; unknown genus; species 486 & $<0.03$ & 个 \\
\hline Bacteroides; species 293 & $<0.03$ & $\downarrow$ \\
\hline Desulfovibrionaceae; unknown genus; species 508 & $<0.05$ & 个 \\
\hline Enterobacteriaceae; unknown genus ;species 217 & $<0.05$ & $\uparrow$ \\
\hline \multicolumn{3}{|l|}{ 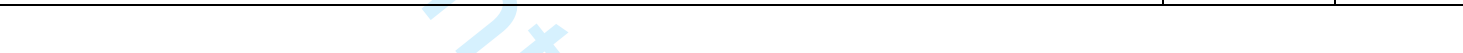 } \\
\hline \multicolumn{3}{|c|}{ Changes post-treatment from treatment phase } \\
\hline TAXA & $p^{1}$ & Trend \\
\hline Streptococcus; species 72 & $<0.01$ & $\uparrow$ \\
\hline Holdemania; species 509 & $<0.01$ & $\downarrow$ \\
\hline Erysipelotrichaceae; genus unknown ;species 27 & $<0.03$ & 个 \\
\hline Clostridiales; genus unknown; species 122 & $<0.03$ & 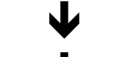 \\
\hline Barnesiellaceae; genus unknown; species 314 & $<0.03$ & $\downarrow$ \\
\hline Clostridiales; genus unknown;species 292 & $<0.03$ & $\downarrow$ \\
\hline Clostridiales; genus unknown; species 251 & $<0.05$ & $\downarrow$ \\
\hline Lachnospiraceae; genus unknown;species 558 & $<0.05$ & $\downarrow$ \\
\hline Enterobacteriaceae; genus unknown; species 449 & $<0.05$ & 个 \\
\hline Bifidobacterium genus & $<0.05$ & $\uparrow$ \\
\hline
\end{tabular}

${ }^{1}$ Non-parametric repeated measures Friedman test. 
Table 3. Changes in bacterial taxa during and after intervention in the prebiotic group

\begin{tabular}{|c|c|c|}
\hline \multicolumn{3}{|c|}{ Changes during treatment from pre-treatment phase } \\
\hline TAXA & $p^{1}$ & Trend \\
\hline Parabacteroides distasonis & $<0.01$ & $\downarrow$ \\
\hline Clostridiaceae; genus unknown; species 319 & $<0.02$ & $\downarrow$ \\
\hline Ruminococcaceae ;genus unknown; species 118 & $<0.02$ & $\uparrow$ \\
\hline Lachnospiraceae; genus unknown; species 40 & $<0.02$ & $\uparrow$ \\
\hline Oscillospira; species 52 & $<0.02$ & $\downarrow$ \\
\hline Oscillospira; species 409 & $<0.03$ & $\downarrow$ \\
\hline Lachnospiraceae; genus unknown; species 783 & $<0.03$ & $\uparrow$ \\
\hline Barnesiellaceae; genus unknown; species 542 & $<0.05$ & $\downarrow$ \\
\hline Christensenellaceae ; genus unknown; species 497 & $<0.05$ & $\downarrow$ \\
\hline Ruminococcus; species 584 & $<0.05$ & $\downarrow$ \\
\hline Bilophila wadsworthia & $<0.05$ & $\boldsymbol{\downarrow}$ \\
\hline
\end{tabular}

\begin{tabular}{l|c|c}
\hline \multicolumn{3}{c}{ Changes post-treatment from treatment phase } \\
\hline \multicolumn{1}{c}{ TAXA } & $\boldsymbol{p}^{\mathbf{1}}$ & Trend \\
\hline Lachnospiraceae; genus unknown; species 19 & $<0.01$ & $\downarrow$ \\
Bacteroides; species 119 & $<0.02$ & $\boldsymbol{\uparrow}$ \\
Clostridiales; genus unknown; species 171 & $<0.02$ & $\downarrow$ \\
Peptococcaceae; genus unknown; species 373 & $<0.03$ & $\downarrow$ \\
Veillonellaceae; genus unknown; species 284 & $<0.03$ & $\boldsymbol{\uparrow}$ \\
Lachnospiraceae; genus unknown; species 206 & $<0.03$ & $\boldsymbol{\uparrow}$ \\
Bacteroides; species 124 & $<0.05$ & $\uparrow$ \\
Clostridiales; genus unknown; species 410 & $<0.05$ & $\downarrow$ \\
Ruminococcaceae; genus unknown; species 130 & $<0.05$ & $\uparrow$ \\
Ruminococcaceae; genus unknown; species 592 & $<0.05$ & $\uparrow$ \\
Alphaproteobacteria; genus unknown; species 223 & $<0.05$ & $\downarrow$ \\
\hline
\end{tabular}

${ }^{1}$ Non-parametric repeated measures Friedman test. 


\section{Figure 1}

10

11

12

13

14

15

16

17

18

19

20

21

22

23

24

25

26

27

28

29

30

31

32

33

34

35

36

37

38

39

40

41

42

43

44

45

46

47

48

49

50

51

52

53

54

55

56

57

58

59

60

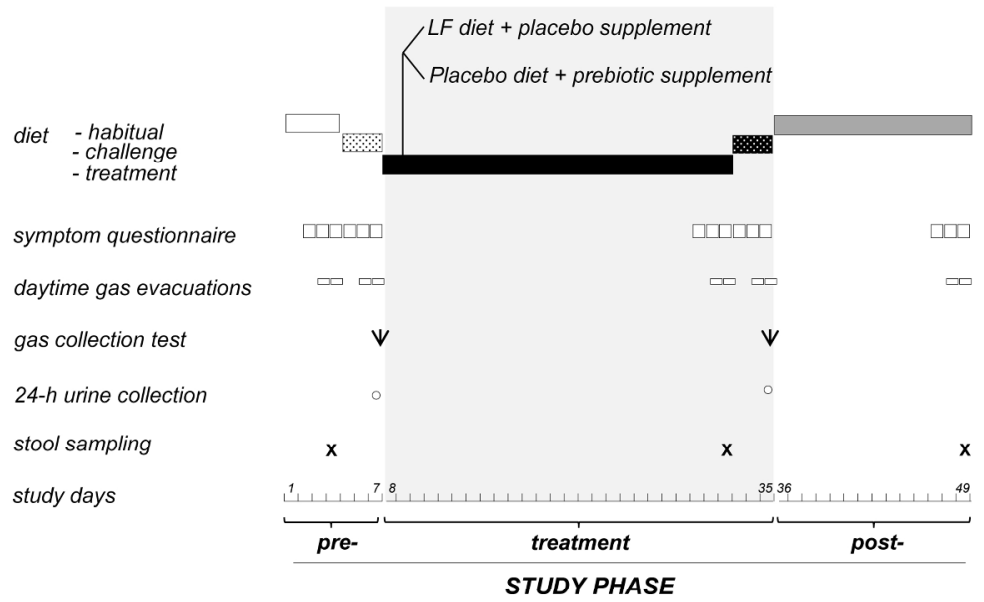

Experimental design. Note the color code: pre-treatment phase white, treatment black and post-treatment grey.

$190 \times 254 \mathrm{~mm}(300 \times 300$ DPI $)$

https://mc.manuscriptcentral.com/gut 
Figure 2

Flow chart

$190 \times 254 \mathrm{~mm}(300 \times 300 \mathrm{DPI})$ 


\section{Figure 3}
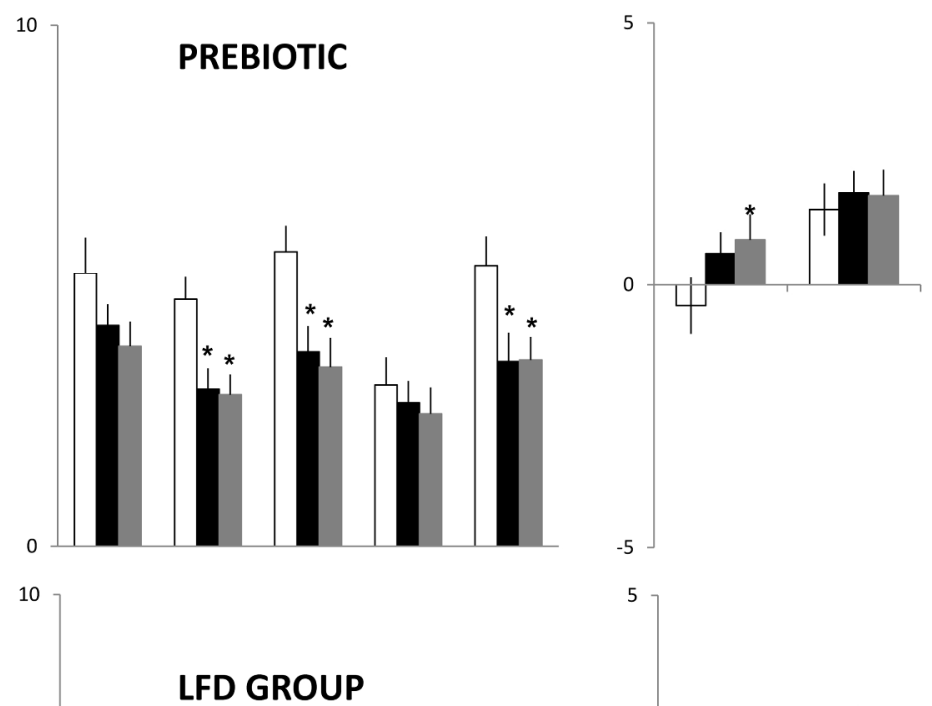

${ }^{*} p<0.05$ vs pre-treatment phase; $+p \leq 0.053$ vs treatment phase

Baseline symptoms measured by daily questionnaires pre-treatment (days 2-4, white), in the treatment phase (days 30-32, black) and in the post-treatment phase (day 47-49, grey) (LFD $n=21$; prebiotic group $\mathrm{n}=19)$. Data are average over each 3-day periods.

$190 \times 254 \mathrm{~mm}(300 \times 300 \mathrm{DPI})$ 
Figure 4

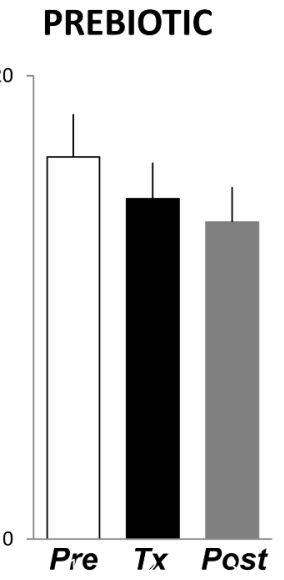

LFD GROUP

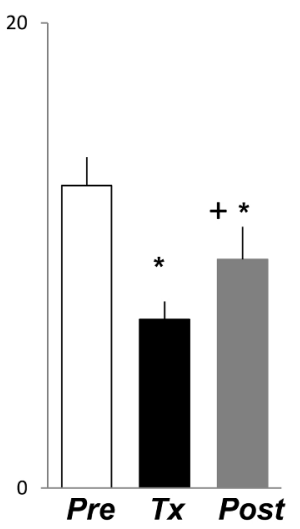

* $p<0.05$ vs pre-treatment phase;

$+p=0.056$ vs treatment

Number of daytime anal gas evacuations during the last 2 days of each evaluation phase: pre-treatment (Pre: days 3-4, white), treatment (Tx: days 31-32, black) and post-treatment (Post: days 48-49, grey) (LFD $\mathrm{n}=21$; prebiotic group $\mathrm{n}=19$ ).

$190 \times 254 \mathrm{~mm}(300 \times 300 \mathrm{DPI})$ 
Figure 5
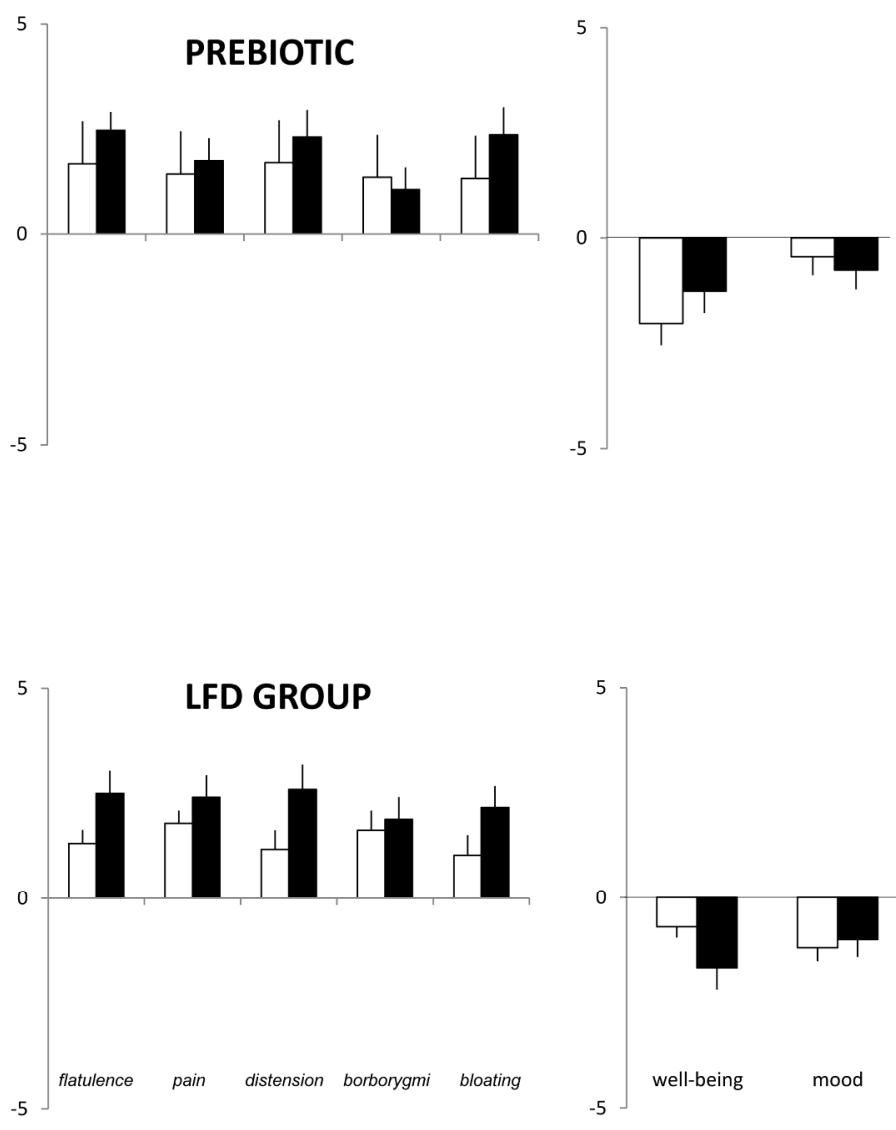

Effect of challenge diet on symptoms measured by daily questionnaires pre-treatment (days 5-7, white) and in the treatment phase (days 33-35, black) (LFD $n=21$; prebiotic group $n=19$ ). Data are average over each 3-day periods. In each group the change during treatment from pre-treatment phase is shown.

$190 \times 254 \mathrm{~mm}(300 \times 300$ DPI $)$ 
Figure 6

Symptoms in response to probe meal in the pre-treatment (day 5 , white) and in the treatment phase (day 33, black) (LFD $n=21$; prebiotic group $n=19$ ).
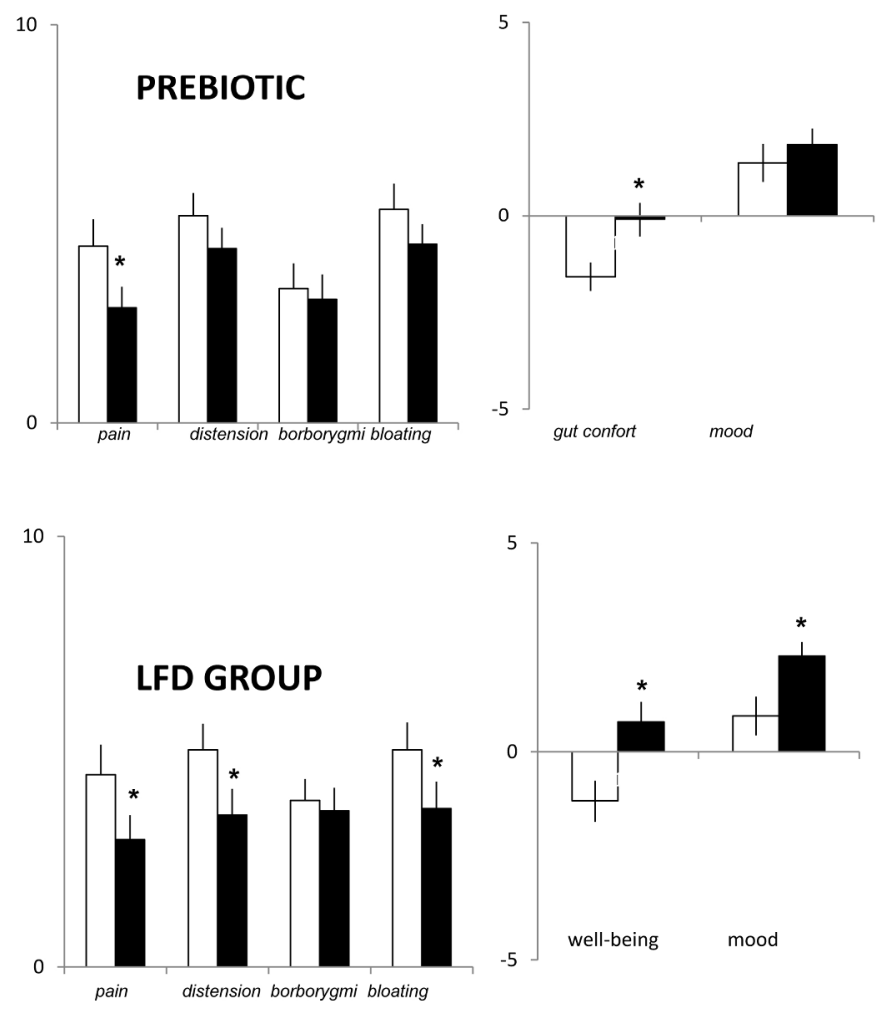

\author{
${ }^{*} p<0.05$ vs pre-treatment phase
}

\author{
$190 \times 254 \mathrm{~mm}(300 \times 300$ DPI $)$
}


Figure 7
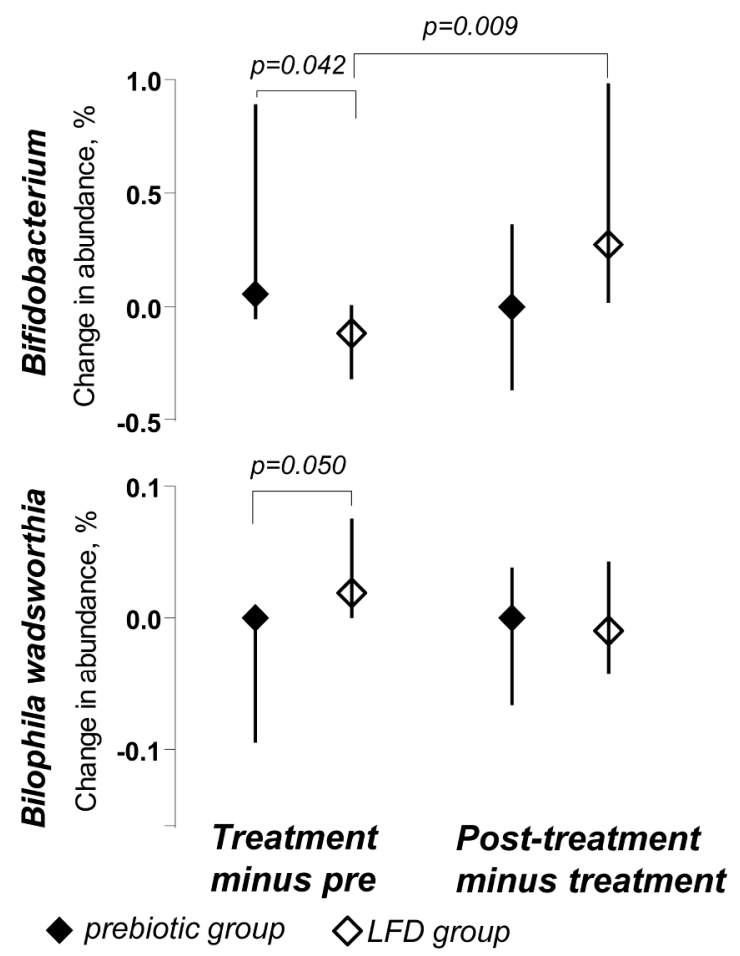

Effect of treatment on relative abundance in Bifidobacterium and Bilophila wadsworthia. Figure shows changes during treatment (from pre-treatment phase) and after treatment (from treatment phase). Overall differences were tested by non-parametric Kruskal-Wallis analysis of variance on ranks, and the MannWhitney test was used for post-hoc comparisons (LFD $n=21$; prebiotic group $n=19$ ). Data are median and interquartile range.

$190 \times 254 \mathrm{~mm}(300 \times 300 \mathrm{DPI})$ 
Supplemental material: microbiota analysis

Genomic DNA extraction. A frozen aliquot $(250 \mathrm{mg})$ of each sample was suspended in $250 \mu \mathrm{L}$ of guanidine thiocyanate, $0.1 \mathrm{M}$ Tris $(\mathrm{pH} 7.5), 40 \mu \mathrm{L}$ of $10 \% \mathrm{~N}$-lauroyl sarcosine and $500 \mu \mathrm{L}$ of $5 \% \mathrm{~N}$-lauroyl sarcosine. DNA was extracted by mechanical disruption of microbial cells with beads, and the recovery of nucleic acids from the clear lysates was achieved by alcohol precipitation, as previously described ${ }^{1}$. An equivalent of $1 \mathrm{mg}$ of each sample was used for DNA quantification using a NanoDrop ND-1000 spectrophotometer (Nucliber, Madrid, Spain).

High-throughput DNA sequencing. To profile the microbiome composition, the hyper-variable region (V4) of the bacterial and archaeal 16S rRNA gene was amplified by PCR. On the basis of our analysis performed using PrimerProspector software ${ }^{2}$, the V4 primer pairs used in this study were expected to amplify almost $100 \%$ of the bacterial and archaeal domains. The 5 ' ends of the forward (V4F_515_19: 5'-GTGCCAGCAMGCCGCGGTAA -3') and reverse (V4R_806_20: 5'- GGACTACCAGGGTATCTAAT -3') primers targeting the 16S gene were tagged with specific sequences as follows: 5'\{AATGATACGGCGACCACCGAGATCTACACTATGGTAATTGT\} \{GTGCCAGCMGCCGCGGTAA\}-3' and 5'\{CAAGCAGAAGACGGCATACGAGAT\} \{Golay barcode\} \{AGTCAGTCAGCC\} \{GGACTACHVGGGTWTCTAAT\}-3'. Multiplex identifiers, known as Golay codes, had 12 bases and were specified downstream of the reverse primer sequence (V4R_806_20) ${ }^{3}$. Standard PCR (0.75 units of Taq polymerase (Roche, Barcelona, Spain) and $20 \mathrm{pmol} / \mu \mathrm{L}$ forward and reverse primers) was run in a Mastercycler gradient (Eppendorf, Madrid, Spain) at $94^{\circ} \mathrm{C}$ for $3 \mathrm{~min}$, 
followed by 35 cycles at $94^{\circ} \mathrm{C}$ for $45 \mathrm{sec}, 56^{\circ} \mathrm{C}$ for $60 \mathrm{sec}, 72^{\circ} \mathrm{C}$ for $90 \mathrm{sec}$, and a final cycle of $72^{\circ} \mathrm{C}$ for $10 \mathrm{~min}$. Amplicons were purified using a QIAquick PCR Purification Kit (Qiagen, Barcelona, Spain), quantified using a NanoDrop ND1000 spectrophotometer (Nucliber, Madrid, Spain), and then pooled in equal concentrations. Pooled amplicons (2 $\mathrm{nM})$ were then subjected to sequencing using Illumina MiSeq technology in the technical support unit of the Autonomous University of Barcelona (UAB, Spain) following standard Illumina platform protocols.

Sequence analysis. Sequences obtained from the 60 faecal samples after the sequencing step were analysed with QIIME (Quantitative Insights into Microbial Ecology)

1.9.1 ${ }^{4}$ using an in-house script that performs upstream and downstream analyses. Low-quality raw sequences with a Phred score of less than 20 were removed from the analysis. Each read was assigned back to its corresponding sample during a demultiplexing step, and the barcodes were removed from the sequences. After filtering, we obtained a total of $2,460,589$ high-quality sequences. The USEARCH (ultra-fast sequence analysis) ${ }^{5}$ tool was used to cluster similar sequences into operational taxonomic units (OTUs) or taxa based on a $97 \%$ similarity level, and the UCHIME (ultra-fast chimeric search) algorithm was used to remove chimeric sequences. From each of the OTUs, one representative sequence was selected and then aligned using PyNAST (Python Nearest Alignment Space Termination tool) against a Greengenes template alignment from the most recent version of the database (gg_13_8). Then, a taxonomical assignment step was performed using the basic local alignment search tool (BLAST) to map each representative sequence against a 
combined database encompassing the Greengenes and PATRIC (Pathosystems Resource Integration Center) databases. A phylogenetic tree was constructed using the FastTree programme and an OTU table. To avoid false positive OTUs, we eliminated those that did not represent at least $0.2 \%$ of the sequences in at least two samples. The final OTU table was rarefied at 15396 sequence reads per sample. Rarefaction is used to overcome cases in which read counts were not similar between samples.

Quantification of Bifidobacterium. To quantify Bifidobacterium spp., the extracted genomic DNA was used to amplify the 16S rRNA gene by quantitative real-time PCR (qPCR) using the following specific primers: Bifgenus_F (5'-TGG CTC AGG ATG AAC GCT G-3'), Bifgenus_R (5'-TGA TAG GAC GCG ACC CCA T-3') and the TaqMan MGB probe $\left(\mathrm{FAM}^{\mathrm{TM}}\right.$ dye-labelled; 5'-CAT CCG GCA TTA CCA-3'). To calibrate the qPCR reactions, we used calculated amounts of extracted DNA from three isolated Bifidobacterium species (B. breve, B. longum and $B$. infantis). Serial dilutions of the pooled DNA were amplified (copy number ranging from 25 to $2.5 \times 10^{6}$ ) to extrapolate the bifidobacterial number in each sample. The qPCR was performed with the 7500 Fast Real-Time PCR system (Applied Biosystems, Barcelona, Spain) using optical-grade 96-well plates. The PCR reaction was performed in a total volume of $25 \mu \mathrm{L}$ using the TaqMan Universal PCR Master Mix (Applied Biosystems), containing $300 \mathrm{nM}$ of each primer and $100 \mathrm{nM}$ of the MGB probe. The reaction conditions for the amplification of DNA were $50^{\circ} \mathrm{C}$ for $2 \mathrm{~min}, 95^{\circ} \mathrm{C}$ for $10 \mathrm{~min}, 40$ cycles of $95^{\circ} \mathrm{C}$ for $15 \mathrm{sec}$ and $60^{\circ} \mathrm{C}$ for $1 \mathrm{~min}$. All reactions were performed in triplicate, and the mean values were calculated. The data were analysed using Sequence Detection Software version 1.4, supplied by Applied Biosystems. 


\section{References}

1. Cardona S, Eck $A$, Cassellas $M$, et al. Storage conditions of intestinal microbiota matter in metagenomic analysis. BMCMicrobiol 2012;12:158.

2. Walters WA, Caporaso JG, Lauber CL. Primer-Prospector: de novo design and taxonomic analysis of barcoded polymerase chain reaction primers. Bioinformatics 2011;27:1159-61.

3. Caporaso JG, Lauber CL, Walters WA, et al. Ultra-high-throughput microbial community analysis on the Illumina HiSeq and MiSeq platforms. ISMEJ 2012;6(8):1621-24.

4. Navas-Molina JA, Peralta-Sanchez JM, Gonzalez A, et al. Advancing our understanding of the human microbiome using QIIME. Methods Enzymol 2013;531:371-444.

5. Edgar RC. Search and clustering orders of magnitude faster than BLAST. Bioinformatics 2010;26(19):2460-61. 